\title{
Evaluating the impact of a brief Health at Every Size-based activity on body positivity and internal weight-based oppression
}

Hanaa Saeed Ahmed \& Sana Ahmad Elashie, Undergraduate Public Health students, Qatar University Dr Lily O'Hara, Associate Professor of Public Health, Qatar University

\section{Introduction}

Weight-based oppression, including teasing, harassment, stigma, prejudice and discrimination based on body weight, is a widespread phenomenon. ${ }^{1}$ Weight-based oppression results from both external and internal sources. Internal weight-based oppression is the internalized negative attitudes, values and beliefs people hold about body weight, and is associated with depression, anxiety, body image disturbance, disordered eating, avoidance of physical activity, and increased calorie consumption. ${ }^{2-6}$

The Health at Every Size (HAES) model offers a health promoting approach to internal weight-based oppression. HAES focuses on improving mental and physical health and wellbeing for people of all sizes and encourages people to reorient their behaviors towards increasing intuitive eating, joyful physical activity, and body positivity, and reducing internal weight-based oppression. Brief interventions based on the HAES model have shown some promise in reducing internalized weight stigma and improving body positivity. ${ }^{7}$

Research on strategies to address weight-based opnreccinn and body positivity is absent from the Arab world, inclu

\section{Objective}

The objective of the study was to evaluate the impi activity on body positivity and internal weight-based oppression in female students at Qatar University.

\section{Methods}
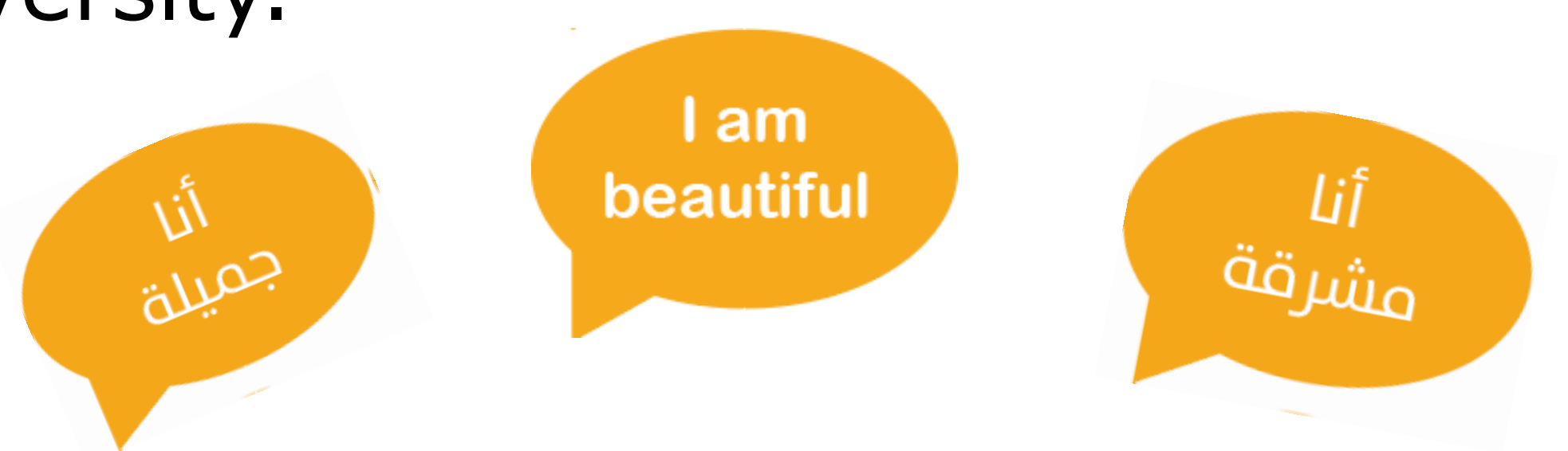

The study used a quasi-experimental pre-post evaluation design, with quantitative assessment (in Arabic and English) of body positivity and internal weight-based oppression before a brief intervention (pre), immediately afterwards (post), and 10 weeks later (follow up).

Four validated scales were used at each time point:

- Body Appreciation Scale 2 (BAS2)

- Modified Weight Bias Internalization Scale (MWBIS)

- Fat Attitudes Assessment Toolkit Self Acceptance subscale (FAAT-SA)

- Fat Attitudes Assessment Toolkit Self Reflection subscale (FAAT-SR)

The brief activity was the Love your Body activity developed and delivered by public health students in the Women's Food Court as part of the Mental Health Festival. The activity involved Yay scales, positive affirmation stickers, photography, postcards, and gratitude writing, and took around 10 to 15 minutes to complete.
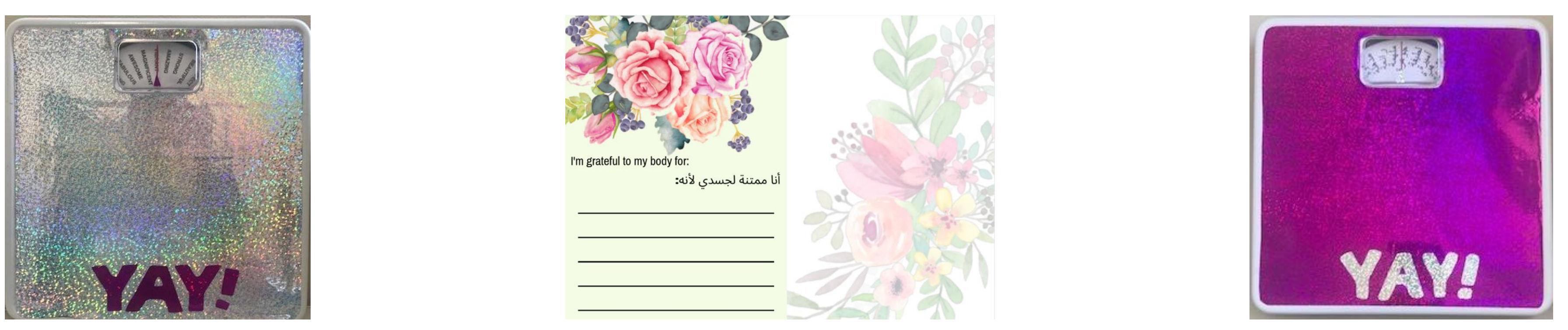

Repeated measures ANOVA tests were used to compare between the different time points, with the Bonferroni correction for multiple comparisons. Statistical Package for the Social Sciences (SPSS) version 26 was used to analyze the data. The study was approved by the QU-IRB, approval number 1145-EA/19.

\section{Results}

A total of 35 female undergraduate students completed the pre, post, and follow up assessments. All measures showed a trend towards improvement from pre to post assessment, but a return to baseline or near baseline status after 10 weeks. Statistically significant changes occurred between the pre and post tests for body appreciation and self reflection. There was a high number of missing values within each scale, reducing the number of complete responses for all three time points to $9-11$ depending on the scale.

7

6

5 Self reflection

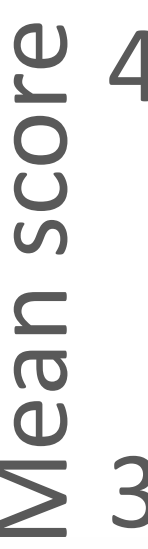

Body appreciation internalisation

\section{كلية العلوم الصحية \\ College of Health Sciences QATAR UNIVERSITY, جاهعة قط of HEALTH تصوفي ألصحـة}

0

Pre

Follow up

$* p=0.008$ for Pre to Post, ${ }^{* *} p=0.001$ for Pre to Post
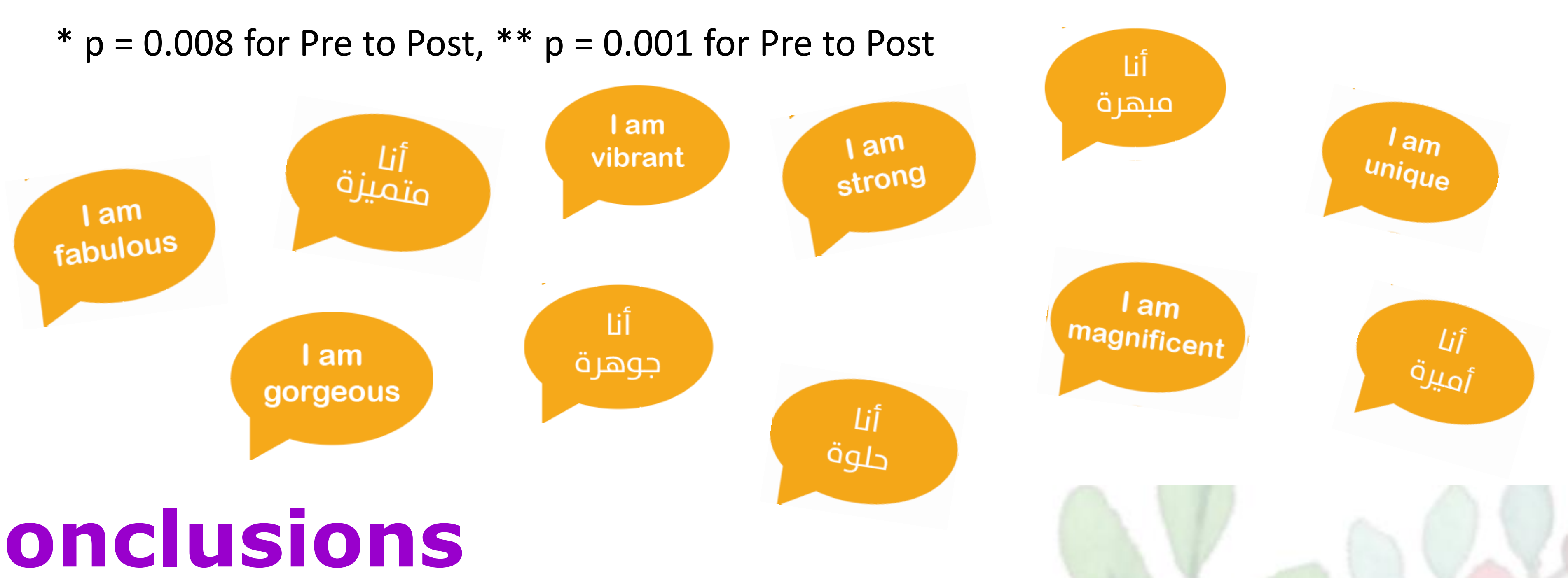

\section{Conclusions}

The Health at Every Size-based activity had a positive effect on participants' body appreciation and self reflection in the short term, but these improvements were not sustained over the longer term. The high number of missing responses may have compromised the potential to determine more robust findings. Further research is required to assess the impact of brief activities on body appreciation and internal weight-based oppression.

\section{References}

1.Puhl, R.M. and C.A. Heuer, The Stigma of Obesity: A Review and Update. Obesity, 2009. 17(5): p. 941-964.

2.Davison, K.K., et al., Overweight Girls Who Internalize Fat Stereotypes Report Low Psychosocial Well-being. Obesity, 2008. 16(S2): p. S30-S38.

3.Wang, S., K. Brownell, and T. Wadden, The influence of the stigma of obesity on overweight individuals. International Journal of Obesity and Related Disorders, 2004. 28(10): p. 1333.

4.Friedman, K.E., J.A. Ashmore, and K.L. Applegate, Recent Experiences of Weight-based Stigmatization in a Weight Loss Surgery Population: Psychological and Behavioral Correlates. Obesity, 2008. 16(S2): p. S69-S74.

5.Puhl, R.M., C.A. Moss-Racusin, and M.B. Schwartz, Internalization of Weight Bias: Implications for Binge Eating and Emotional Well-being. Obesity, 2007. 15(1): p. 19-23. 